UWThPh-1996-5

August 30, 1996

\title{
The Luttinger-Schwinger Model
}

\author{
Harald Grosse*, Edwin Langmann** and Ernst Raschhofer*, \\ * Institut für Theoretische Physik, Universität Wien, A-1090 Wien, Austria \\ ** Theoretical Physics, Royal Institute of Technology, S-10044 Stockholm, Sweden
}

\begin{abstract}
We study the Luttinger-Schwinger model, i.e. the $(1+1)$ dimensional model of massless Dirac fermions with a non-local 4-point interaction coupled to a U(1)-gauge field. We work within the Hamiltonian framework on the cylinder, and construct the field operators and observables as well-defined operators on the physical Hilbert space. The complete solution of the model is found using the boson-fermion correspondence, and the formalism for calculating all gauge invariant Green functions is provided. We discuss the role of anomalies and show how the existence of large gauge transformations implies a fermion condensate in all physical states. The meaning of regularization and renormalization in our well-defined Hilbert space setting is discussed. We illustrate the latter by performing the limit to the Thirring-Schwinger model where the interaction becomes local.
\end{abstract}

\footnotetext{
${ }^{a}$ Supported in part by the "Fonds zur Förderung von Wissenschaft und Forschung in Österreich" under contract P8916-PHY
} 


\section{Introduction}

Since the early days of quantum field theory (QFT) $1+1$ dimensional models have attracted much attention. They have been extremely valuable to develop general ideas and intuition about the structure of QFT. The eldest and perhaps most popular of these $1+1 \mathrm{D}$ models bear the names of Thirring [1] - Dirac fermions interacting with a local current-current interaction - and Schwinger [2] - quantum electrodynamics with fermions. The models originated in particle physics and therefore, in order to have Lorentz invariance, were considered mainly on infinite space $\mathbb{R}$ (see e.g. [3, 4, 5] and references therein). Then one has to deal with infrared (infinite space volume) divergences in addition to singularities coming from the ultraviolet (short distances). In case the fermions are massless, both models are soluble [2, 6, 7] and a very detailed picture of their properties can be obtained. Another related model originated from solid state physics and is due to Luttinger [8] - massless Dirac fermions on spacetime $S^{1} \times \mathbb{R}$ interacting with a non-local current-current interaction (Lorentz invariance is nothing natural to ask for in solid state physics). The Luttinger model shows that an interacting fermion system in one space dimension need not behave qualitatively similar to free fermions but rather has properties similar to a boson system. Such behaviour is generic for $1+1 \mathrm{D}$ interacting fermion models and is denoted as Luttinger liquid in solid state physics, in contrast to Landau liquids common in $3+1 \mathrm{D}$.

To consider the Luttinger model on compact space has the enormous technical advantage that infra red (IR) problems are absent, and one can concentrate on the short distance (UV) properties which are rather simple due to the non-locality of the interaction. In fact, this allows a construction of the interacting model on the Fock space of free fermions [9, 10, 11] and one directly can make use of mathematical results from the representation theory of the affine Kac-Moody algebras. Such an approach was recently given for QCD with massless fermions [12]. As shown by Manton [13], the Schwinger model on compact space $S^{1}$ allows a complete understanding of the UV divergences and anomalies and their intriguing interplay with gauge invariance and vacuum structure.

In the present paper we study the extension of the Luttinger model obtained by coupling it to a dynamical electromagnetic field. For vanishing Luttinger (4-point) interaction our model therefore reduces to the Schwinger model as studied by Manton [13], and for vanishing electric charge to the Luttinger model [9]. Since our approach is in Minkowski space and provides a direct construction of the field- and observable algebras of the model on a physical Hilbert space, it is conceptually quite different from the path integral approach, and we believe it adds to the physical understanding of these models.

The plan of the paper is as follows. In Section 2 the construction of the model is given. To fix notation, we first summarize the classical Hamiltonian formalism. We then construct the physical Hilbert space and discuss the non-trivial implications of anomalies (Schwinger terms) and gauge invariance. In Section 3 the model is solved by bosonization, and a method for calculating all Green functions is explained. As an example the equal time 2point functions are given. In Section 4 we comment on regularization and renormalization in our setting. We discuss the limit to the Thirring-Schwinger model where the 4-point interaction becomes local and space infinite. We end with a short summary in Section 5. A summary of the mathematical results needed and some details of calculations are deferred to the appendix. 


\section{Constructing the model}

\section{$2.1 \quad$ Notation}

Spacetime is the cylinder with $x=x^{1} \in \Lambda \equiv[-L / 2, L / 2]$ the spatial coordinate and $t=x^{0} \in \mathbb{R}$ time. We have one Dirac Fermion field $\psi_{\sigma}(\boldsymbol{x})$ and one Photon field $A_{\nu}(\boldsymbol{x})$ (here and in the following, $\sigma, \sigma^{\prime} \in\{+,-\}$ are spin indices, $\mu, \nu \in\{0,1\}$ are spacetime indices, and $\boldsymbol{x}=(t, x), \boldsymbol{y}=\left(t^{\prime}, y\right)$ are spacetime arguments).

The action defining the Luttinger-Schwinger model ist?

$$
\begin{array}{r}
\mathcal{S}=\int d^{2} \boldsymbol{x}\left(-\frac{1}{4} F_{\mu \nu}(\boldsymbol{x}) F^{\mu \nu}(\boldsymbol{x})+\bar{\psi}(\boldsymbol{x}) \gamma^{\nu}\left(-\mathrm{i} \partial_{\nu}+e A_{\nu}(\boldsymbol{x})\right) \psi(\boldsymbol{x})\right) \\
-\int d^{2} \boldsymbol{x} \int d^{2} \boldsymbol{y} j_{\mu}(\boldsymbol{x}) v(\boldsymbol{x}-\boldsymbol{y}) j^{\mu}(\boldsymbol{y})
\end{array}
$$

where $F_{\mu \nu}=\partial_{\mu} A_{\nu}-\partial_{\nu} A_{\mu}$ and $\gamma^{\nu} \equiv\left(\gamma^{\nu}\right)_{\sigma \sigma^{\prime}}$ are Dirac matrices which we take as $\gamma^{0}=\sigma_{1}$ and $\gamma^{1}=\mathrm{i} \sigma_{2}$, and $\gamma_{5}=-\gamma^{0} \gamma^{1}=\sigma_{3}$ ( $\sigma_{i}$ are Pauli spin matrices). As usual, the fermion currents are $j_{\nu}=\bar{\psi} \gamma_{\nu} \psi$, and we assume the 4 -point interaction to be instantaneous (local in time)

$$
v(\boldsymbol{x}-\boldsymbol{y})=\delta\left(t-t^{\prime}\right) V(x-y)
$$

where the interaction potential is parity invariant, $V(x)=V(-x)$. As in case of the Luttinger model [10] we will also have to assume that this potential is not 'too strong', or more precisely that the Fourier coefficients

$$
W_{k}=\frac{1}{8 \pi} \int_{\Lambda} \mathrm{d} x V(x) \mathrm{e}^{-\mathrm{i} k x}=W_{-k}=W_{k}^{*}, \quad k \frac{L}{2 \pi} \in \mathbb{Z}
$$

of the potential obey the conditions

$$
-1-\frac{e^{2}}{\pi k^{2}}<W_{k}<1 \quad \forall k \quad \text { and } \quad \sum_{k}\left|k W_{k}^{2}\right|<\infty .
$$

From the action (11) we obtain the canonical momenta $\Pi_{A_{0}(x)} \simeq 0, \Pi_{A_{1}(x)}=F_{01}(x)=$ $E(x)$ etc. (here and in the following, we set $t=0$ and make explicit the dependence on the spatial coordinate only) resulting in the Hamiltonian $\left(\psi^{*} \equiv \bar{\psi} \gamma^{0}\right)$

$H=\int_{\Lambda} \mathrm{d} x\left(\frac{1}{2} E(x)^{2}+\psi^{*}(x) \gamma_{5}\left(-\mathrm{i} \partial_{1}+e A_{1}(x)\right) \psi(x)\right)+4 \int_{\Lambda} \mathrm{d} x \mathrm{~d} y \rho^{+}(x) V(x-y) \rho^{-}(y)$,

and the Gauss' law

$$
G(x)=-\partial_{1} E(x)+e \rho(x) \simeq 0 .
$$

We introduced chiral fermion currents

$$
\rho^{ \pm}(x)=\psi^{*}(x) \frac{1}{2}\left(1 \pm \gamma_{5}\right) \psi(x)
$$

so that fermion charge- and momentum density $\rho=j^{0}$ and $j=j^{1}$ can be written as

$$
\begin{aligned}
& \rho(x)=\rho^{+}(x)+\rho^{-}(x) \\
& j(x)=\rho^{+}(x)-\rho^{-}(x) .
\end{aligned}
$$

\footnotetext{
${ }^{1}$ unless otherwise stated, repeated indices are summed over throughout the paper

${ }^{2} \partial_{\nu} \equiv \partial / \partial x^{\nu}$; our metric tensor is $g_{\mu \nu}=\operatorname{diag}(1,-1)$
} 


\subsection{Observables}

The observables of the model are all gauge invariant operators. They leave invariant physical states. The ground state expectation values of these operators are the Green functions we are interested in. For later reference we write down the action of static gauge transformations i.e. differentiable maps $\Lambda \rightarrow \mathrm{U}(1), x \mapsto \mathrm{e}^{\mathrm{i} \alpha(x)}$,

$$
\begin{aligned}
\psi_{\sigma}(x) & \rightarrow \mathrm{e}^{\mathrm{i} \alpha(x)} \psi_{\sigma}(x) \\
A_{1}(x) & \rightarrow A_{1}(x)-\frac{1}{e} \frac{\partial \alpha(x)}{\partial x} \\
E(x) & \rightarrow E(x)
\end{aligned}
$$

These obviously leave our Hamiltonian and Gauss' law invariant. We note that every gauge transformation can be decomposed into a small and a large gauge transformation, $\alpha(x)=\alpha_{\text {small }}(x)+\alpha_{\text {large }}(x)$, where

$$
\alpha_{\text {large }}(x)=n \frac{2 \pi x}{L} \quad(n \in \mathbb{Z}), \quad \alpha_{\text {small }}\left(-\frac{L}{2}\right)=\alpha_{\text {small }}\left(\frac{L}{2}\right)
$$

with $n=\frac{\alpha(L / 2)-\alpha(-L / 2)}{2 \pi L}$. The large gauge transformations correspond to $\Pi_{1}\left(S^{1}\right)=\mathbb{Z}$ and play an important role in the following, as expected from general arguments [14]. It is important to note that Gauss' law (5) requires physical states only to be invariant under small (but not under large) gauge transformations.

All gauge invariant objects which one can construct from $A_{1}(x)$ (at fixed time) are functions of

$$
Y=\frac{1}{2 \pi} \int_{\Lambda} \mathrm{d} y A_{1}(y)
$$

In fact, $Y$ above is only invariant with respect to small gauge transformations and changes by multiples of $1 / e$ under the large ones. Thus the quantity which is invariant under all gauge transformations is $\mathrm{e}^{\mathrm{i} 2 \pi e Y}$ which is equal to the Wilson line (holonomy)

$$
W\left[A_{1}\right]=\mathrm{e}^{\mathrm{i} e \int_{\Lambda} \mathrm{d} y A_{1}(y)} .
$$

The fermion fields are not gauge invariant, but by attaching parallel transporters to them one obtains field operators

$$
\chi_{\sigma}(x)=\mathrm{e}^{\mathrm{i} e \int_{r}^{x} \mathrm{~d} y A_{1}(y)} \psi_{\sigma}(x), \quad r \in \Lambda
$$

which obviously are invariant under all (small and large) gauge transformations (8) with $\alpha(r)=0 ; r$ is a spatial point which we can choose arbitrarily. Note that these fields also obey CAR but are not antiperiodic: they obey $\chi_{\sigma}(L / 2)=-W\left[A_{1}\right] \chi_{\sigma}(-L / 2)$ where $W\left[A_{1}\right]$ is the Wilson line above. Bilinears of these operators are the meson operators

$$
M_{\sigma \sigma^{\prime}}(x, y)=\chi_{\sigma}^{*}(x) \chi_{\sigma^{\prime}}(y)
$$

These are invariant under all static gauge transformations and thus can be used as building blocks of the Green functions we are interested in. 


\subsection{The quantum model}

In the following we find it convenient to work in Fourier space. We introduce the following useful notation. Fourier space for even (periodic) functions is

$$
\Lambda^{*} \equiv\left\{k=\frac{2 \pi}{L} n \mid n \in \mathbb{Z}\right\} .
$$

As we use fermions with odd (anti-periodic) boundary conditions we also need

$$
\Lambda_{\text {odd }}^{*} \equiv\left\{k=\frac{2 \pi}{L}\left(n+\frac{1}{2}\right) \mid n \in \mathbb{Z}\right\} .
$$

For functions $\hat{f}$ on Fourier space we write

$$
\hat{\int}_{\Lambda^{*}} \hat{\mathrm{d}} k \hat{f}(k) \equiv \sum_{k \in \Lambda^{*}} \frac{2 \pi}{L} \hat{f}(k)
$$

and similarly for $\Lambda_{\text {odd }}^{*}$ (we will use the same symbols $\delta$ and $\hat{\delta}$ also in the latter case). Then the appropriate $\delta$-function satisfying $\hat{\int}_{\Lambda^{*}} \hat{\mathrm{d}} q \hat{\delta}(k-q) \hat{f}(q)=\hat{f}(k)$ is $\hat{\delta}(k-q) \equiv \frac{L}{2 \pi} \delta_{k, q}$.

For the Fourier transformed operators we use the following conventions,

$$
\hat{\psi}_{\sigma}(q)=\int_{\Lambda} \frac{\mathrm{d} x}{\sqrt{2 \pi}} \psi_{\sigma}(x) \mathrm{e}^{-\mathrm{i} q x}, \quad \hat{\psi}_{\sigma}^{*}(q)=\hat{\psi}_{\sigma}(q)^{*} \quad\left(q \in \Lambda_{o d d}^{*}\right)
$$

(as mentioned, we use anti-periodic boundary conditions for the fermions),

$$
\hat{A}_{1}(k)=\int_{\Lambda} \frac{\mathrm{d} x}{2 \pi} A_{1}(x) \mathrm{e}^{-\mathrm{i} k x} \quad\left(k \in \Lambda^{*}\right)
$$

and in the other cases

$$
\hat{Y}(k)=\int_{\Lambda} \mathrm{d} x Y(x) \mathrm{e}^{-\mathrm{i} k x} \quad\left(k \in \Lambda^{*}\right) \quad \text { for } Y=E, \rho^{ \pm}, \rho, j, V
$$

Following [10] we also find it convenient to introduce $W_{k}=\hat{V}(k) / 8 \pi$ (cf. (3a)). With that the non-trivial $\mathrm{C}(\mathrm{A}) \mathrm{CR}$ in Fourier space are

$$
\begin{aligned}
{\left[\hat{A}_{1}(p), \hat{E}(k)\right] } & =\mathrm{i} \hat{\delta}(k+p) \\
\left\{\hat{\psi}_{\sigma}(q), \hat{\psi}_{\sigma^{\prime}}^{*}\left(q^{\prime}\right)\right\} & =\delta_{\sigma \sigma^{\prime}} \hat{\delta}\left(q-q^{\prime}\right) .
\end{aligned}
$$

The essential physical requirement determining the construction of the model and implying a non-trivial quantum structure is positivity of the Hamiltonian on the physical Hilbert space. It is well-known that it forces one to use a non-trivial representation of the field operators of the model. The essential simplification in $(1+1)$ (and not possible in higher) dimensions is that one can use a quasi-free representation for the fermion field operators corresponding to "filling up the Dirac sea" associated with the free fermion Hamiltonian, and for the photon operators one can use a naive boson representation. This will be verified for our model for the class of potentials $V$ obeying (3a,b).

So the full Hilbert space of the model is $\mathcal{H}=\mathcal{H}_{\text {Photon }} \otimes \mathcal{H}_{\text {Fermion }}$. For $\mathcal{H}_{\text {Photon }}$ we take the boson Fock space generated by boson field operators $b^{*}(k)$ obeying CCR

$$
\left[b(k), b^{*}(p)\right]=\hat{\delta}(k-q) \quad \text { etc. }
$$


and a vacuum ${ }^{3} \Omega_{\mathrm{P}}$ such that

$$
b(k) \Omega_{\mathrm{P}}=0 \quad \forall k \in \Lambda^{*} .
$$

We then set

$$
\hat{A}_{1}(k)=\frac{1}{s}\left(b(k)+b^{*}(k)\right) \quad \hat{E}(k)=-\frac{\mathrm{i} s}{2}\left(b(k)-b^{*}(k)\right)
$$

where $s^{4}=\pi e^{2}$ (the reason for choosing this factor $s$ will become clear later). We will use below normal ordering $\stackrel{\times}{\times} \ldots \stackrel{\times}{\times}$ of bilinears in the Photon field operators with respect to the vacuum $\Omega_{\mathrm{P}}$, for example $\times$ × $b(k) b^{*}(p) \stackrel{\times}{\times}=b^{*}(p) b(k)$.

For $\mathcal{H}_{\text {Fermion }}$ we take the Fermion Fock space with vacuum $\Omega_{\mathrm{F}}$ such that

$$
\frac{1}{2}\left(1 \pm \gamma_{5}\right) \hat{\psi}( \pm q) \Omega_{\mathrm{F}}=\frac{1}{2}\left(1 \mp \gamma_{5}\right) \hat{\psi}^{*}(\mp q) \Omega_{\mathrm{F}}=0 \quad \forall q>0 .
$$

The presence of the Dirac sea requires normal-ordering : $\cdots$ : of the Fermion bilinears such as $\hat{H}_{0}=\hat{\int}_{\Lambda_{\text {odd }}^{*}} \hat{\mathrm{d}} q: q \hat{\psi}^{*}(q) \gamma_{5} \hat{\psi}(q)$ : and $\hat{\rho}_{ \pm}($(6). This modifies their naive commutator relations following from the CAR as Schwinger terms show up [15, 16, 17]. In our case, the relevant commutators are:

$$
\begin{aligned}
{\left[\hat{\rho}^{ \pm}(k), \hat{\rho}^{ \pm}(p)\right] } & = \pm k \hat{\delta}(k+p) \\
{\left[\hat{\rho}^{ \pm}(k), \hat{\rho}^{\mp}(p)\right] } & =0 \\
{\left[\hat{H}_{0}, \hat{\rho}^{ \pm}(k)\right] } & = \pm k \hat{\rho}^{ \pm}(k) .
\end{aligned}
$$

We note that

$$
\hat{\rho}^{+}(k) \Omega_{\mathrm{F}}=\hat{\rho}^{-}(-k) \Omega_{\mathrm{F}}=0 \quad \forall k>0
$$

which together with (19) shows that the $\hat{\rho}^{+}(k)$ (resp. $\left.\hat{\rho}^{-}(k)\right)$ give a highest (resp. lowest) weight representation of the Heisenberg algebra.

We can now write the Gauss' law operators in Fourier space as

$$
\hat{G}(k)=-\mathrm{i} k \hat{E}(k)+e \hat{\rho}(k),
$$

so eqs. (19) imply

$$
\left[\hat{G}(k), \hat{\rho}^{ \pm}(p)\right]= \pm k e \hat{\delta}(k+p) .
$$

Due to the presence of the Schwinger terms, these Fermion currents no longer commute with the Gauss' law generators, hence they are not gauge invariant and no observables of the model.

To obtain Fermion currents obeying the classical relations (without Schwinger terms), we note that $\left[\hat{G}(k), \hat{A}_{1}(p)\right]=k \hat{\delta}(k+p)$, hence the operators

$$
\tilde{\rho}^{ \pm}(k) \equiv \hat{\rho}^{ \pm}(k) \pm e \hat{A}_{1}(k)
$$

commute with the Gauss law generators and are thus the observables of the model corresponding to the chiral Fermion currents on the quantum level. Recalling the normalization is only unique up to finite terms, it is natural to regard the $\tilde{\rho}^{ \pm}(k)$ as the fermion currents

\footnotetext{
${ }^{3}$ Note that the term "vacuum" here and in the following does not mean that this state has anything to do with the ground state of the model; it is just one convenient state from which all other states in the Hilbert space can be generated by applying the field operators.
} 
obtained by a gauge covariant normal ordering preserving the classical transformation properties under gauge transformations. Indeed, these currents can be shown to be identical to those obtained by the gauge invariant point splitting method.

Similarly, the naive Hamiltonian $\hat{H}=\hat{H}_{1}+\hat{H}_{2}$,

$$
\begin{aligned}
\hat{H}_{1} & =\hat{H}_{0}+\hat{\int}_{\Lambda^{*}} \hat{\mathrm{d}} k \times\left(\frac{1}{4 \pi} \hat{E}(k) \hat{E}(-k)+e \hat{A}_{1}(k) \hat{j}(-k)\right) \times \\
\hat{H}_{2} & =\int_{\Lambda^{*}} \hat{\mathrm{d}} k \hat{\rho}^{+}(k) W_{k} \hat{\rho}^{-}(-k)
\end{aligned}
$$

is not gauge invariant: $\hat{H}_{1}$ - which is the naive Hamiltonian of the Schwinger model - obeys

$$
\left[\hat{G}(k), \hat{H}_{1}\right]=2 k e^{2} \hat{A}_{1}(k)
$$

and therefore becomes gauge invariant only after adding a photon mass term [13]

$$
\hat{\int}_{\Lambda^{*}} \hat{\mathrm{d}} k e^{2} \hat{A}_{1}(k) \hat{A}_{1}(-k)
$$

(note that in position space this mass term has the usual form $\frac{e^{2}}{2 \pi} \int_{\Lambda} \mathrm{d} x A_{1}(x)^{2}$, i.e. the photon mass-squared is $e^{2} / \pi$ ). Also the Luttinger-interaction term $\hat{H}_{2}$ becomes gauge invariant only if one replaces the non-gauge invariant currents $\hat{\rho}^{ \pm}$by the gauge invariant $\tilde{\rho}^{ \pm}$ones.

Thus we obtain the gauge invariant Hamiltonian of the Luttinger-Schwinger model as follows,

$$
\begin{aligned}
H=\hat{H}_{0}+\int_{\Lambda^{*}} \hat{\mathrm{d}} k \times( & \frac{1}{4 \pi} \hat{E}(k) \hat{E}(-k)+e \hat{A}_{1}(k) \hat{j}(-k)+e^{2} \hat{A}_{1}(k) \hat{A}_{1}(-k) \\
& \left.+\left[\hat{\rho}^{+}(k)+e \hat{A}_{1}(k)\right] W_{k}\left[\hat{\rho}^{-}(-k)-e \hat{A}_{1}(-k)\right]\right) \times
\end{aligned}
$$

We can now explain the choice $(17 \mathrm{a}, \mathrm{b})$ for the representation of the Photon field: the factor $s$ is determined such that the free Photon Hamiltonian is equal to $\hat{\int}_{\Lambda^{*}} \hat{\mathrm{d}} k \sqrt{\frac{e^{2}}{\pi}} b^{*}(k) b(k)$.

\subsection{Bosonization}

Kronig's identity allows us to rewrite the free Hamiltonian as $\hat{H}_{0}=\frac{1}{2} \int_{\Lambda^{*}} \hat{\mathrm{d}} k \times{ }^{\times}\left(\hat{\rho}^{+}(k) \hat{\rho}^{+}(-k)\right.$ $\left.+\hat{\rho}^{-}(k) \hat{\rho}^{-}(-k)\right) \times$ (cf. Appendix A for the precise definition of normal ordering; for simplicity of notation we do not distinguish the normal ordering symbol for the photon fields and the fermion currents). With that, it follows from eq. (22) that

$$
H=\int_{\Lambda^{*}} \hat{\mathrm{d}} k \times \times\left(\frac{1}{2}\left(\tilde{\rho}^{+}(k) \tilde{\rho}^{+}(-k)+\tilde{\rho}^{-}(k) \tilde{\rho}^{-}(-k)\right)+\frac{1}{4 \pi} \hat{E}(k) \hat{E}(-k)+\tilde{\rho}^{+}(k) W_{k} \tilde{\rho}^{-}(-k)\right) \times
$$

which is now explicitly gauge invariant.

\footnotetext{
${ }^{4}$ in the modern literature this is often referred to as (special case of the) Sugawara construction
} 


\section{Solution of the model}

\subsection{Gauge Fixing}

The only gauge invariant degree of freedom of the Photon field at fixed time is the holonomy $\int_{\Lambda} \mathrm{d} x A_{1}(x)$ and one can gauge away all Fourier modes $\hat{A}_{1}(k)$ of the gauge field except the one for $k=0$. Thus we can impose the gauge condition

$$
\hat{A}_{1}(k)=\delta_{k, 0} Y, \quad A_{1}(x)=\frac{2 \pi}{L} Y
$$

and solve the Gauss' law $\hat{G}(k) \simeq 0$ (cf. eq. (21)) as

$$
\hat{E}(k) \simeq \frac{e \hat{\rho}(k)}{\mathrm{i} k} \text { for } k \neq 0
$$

This determines all components of $E$ except those conjugate to $Y: \hat{E}(0)=\frac{L}{2 \pi} \frac{\partial}{\mathrm{i} \partial Y}$. After that we are left with the $(k=0)$-component of Gauss' law, viz.

$$
e Q_{0} \simeq 0, \quad Q_{0}=\hat{\rho}(0)=\hat{\rho}^{+}(0)+\hat{\rho}^{-}(0) .
$$

Inserting this into (24), gives the Hamiltonian of the model on the physical Hilbert space $\mathcal{H}_{\text {phys }}=\mathcal{L}^{2}(\mathbb{R}, \mathrm{d} Y) \otimes \mathcal{H}_{\text {Fermion }}^{\prime}$ (where $\mathcal{H}_{\text {Fermion }}^{\prime}$ is the zero charge sector of the fermionic Fock space):

$$
\begin{gathered}
H=-\frac{L}{8 \pi^{2}} \frac{\partial^{2}}{\partial Y^{2}}+\frac{\pi}{L}\left(\left(\hat{\rho}^{+}(0)+e Y\right)^{2}+\left(\hat{\rho}^{-}(0)-e Y\right)^{2}+\left(\hat{\rho}^{+}(0)+e Y\right) 2 W_{0}\left(\hat{\rho}^{-}(0)-e Y\right)\right)+ \\
\int_{\Lambda^{*} \backslash\{0\}} \hat{\mathrm{d}} k \times\left(\frac{e^{2}}{4 \pi k^{2}} \hat{\rho}(k) \hat{\rho}(-k)+\frac{1}{2}\left(\hat{\rho}^{+}(-k) \hat{\rho}^{+}(k)+\hat{\rho}^{-}(k) \hat{\rho}^{-}(-k)\right)+\hat{\rho}^{+}(k) W_{k} \hat{\rho}^{-}(-k)\right) \times
\end{gathered}
$$

\subsection{Diagonalization of the Hamiltonian}

Following [10] we now write

$$
H=\frac{2 \pi}{L} \sum_{k \geq 0} h_{k}
$$

Introducing boson creation- and annihilation operators

$$
c(k)= \begin{cases}\frac{1}{\sqrt{|k|}} \hat{\rho}^{+}(k) & \text { for } k>0 \\ \frac{1}{\sqrt{|k|}} \hat{\rho}^{-}(k) & \text { for } k<0\end{cases}
$$

obeying usual CCR

$$
\left[c(k), c^{*}(p)\right]=\hat{\delta}(k-p) \quad \text { etc. }
$$

We then get for $h_{k>0}$

$$
h_{k}=\left(k+\frac{e^{2}}{2 \pi k}\right)\left(c^{*}(k) c(k)+c^{*}(-k) c(-k)\right)+\left(k W_{k}+\frac{e^{2}}{2 \pi k}\right)\left(c^{*}(k) c^{*}(-k)+c(k) c(-k)\right) \text {. }
$$


For $k=0$ we introduce the quantum mechanical variables

$$
\begin{aligned}
P & =\left(\hat{\rho}^{+}(0)-\hat{\rho}^{-}(0)+2 e Y\right), \\
X & =\mathrm{i} \frac{L}{2 \pi} \frac{1}{2 e} \frac{\partial}{\partial Y}
\end{aligned}
$$

obeying Heisenberg relations, $[P, X]=-\mathrm{i} L / 2 \pi$, which allow us to write $h_{0}$ as Hamiltonian of a harmonic oscillator,

$$
h_{0}=\frac{e^{2}}{\pi} X^{2}+\frac{1}{4}\left(1-W_{0}\right) P^{2}+\frac{1}{4}\left(1+W_{0}\right) Q_{0}^{2}-\frac{1}{2} \sqrt{\frac{e^{2}}{\pi}} \frac{L}{2 \pi}
$$

(the last term stems from normal ordering $\underset{\times}{\times} \ldots \times \times \times$ and is irrelevant for the following).

We can now solve the model by diagonalizing its decoupled Fourier modes $h_{k}$ separately, with the help of a boson Bogoliubov transformation preserving the CCR,

$$
C(k)=\cosh \left(\lambda_{k}\right) c(k)+\sinh \left(\lambda_{k}\right) c^{*}(-k)
$$

where $\lambda_{k}=\lambda_{-k}$. This leads to

$$
h_{k}=\omega_{k}\left(C^{*}(k) C(k)+C^{*}(-k) C(-k)\right)-2 \eta_{k} \frac{L}{2 \pi}
$$

if we choose

$$
\tanh \left(2 \lambda_{k}\right)=\frac{2 \pi k^{2} W_{k}+e^{2}}{2 \pi k^{2}+e^{2}}
$$

Then

$$
\omega_{k}^{2}=k^{2}\left(1-W_{k}^{2}\right)+\frac{e^{2}}{\pi}\left(1-W_{k}\right)
$$

and

$$
\eta_{k}=\frac{1}{2}\left(|k|+\frac{e^{2}}{2 \pi|k|}-\omega_{k}\right) \quad(k \neq 0) .
$$

The zero-momentum piece $h_{0}$ is just a harmonic oscillator and can be written as

$$
h_{0}=\omega_{0} C^{*}(0) C(0)+\frac{1}{4}\left(1+W_{0}\right) Q_{0}^{2}-\eta_{0} \frac{L}{2 \pi}
$$

with

$$
C(0)=\frac{1}{\sqrt{2}}\left(r X+\frac{1}{r} \mathrm{i} P\right), \quad r^{4}=\frac{e^{2}}{\pi} \frac{4}{1-W_{0}},
$$

energy-squared

$$
\omega_{0}^{2}=\frac{e^{2}}{\pi}\left(1-W_{0}\right)
$$

and zero point energy

$$
\eta_{0}=\frac{1}{2}\left(\sqrt{\frac{e^{2}}{\pi}}-\sqrt{\frac{e^{2}}{\pi}\left(1-W_{0}\right)}\right) .
$$

Thus we get the Hamiltonian in the following form

$$
H=\hat{\int}_{\Lambda^{*}} \hat{\mathrm{d}} k \omega_{k} C^{*}(k) C(k)-L E_{0}
$$


with the ground state energy density given by

$$
E_{0}=\frac{1}{2 \pi} \hat{\Lambda^{*}} \hat{\mathrm{d}} k \eta_{k}
$$

(Note that for large $|k|, \eta_{k}=\frac{1}{2}\left(\frac{1}{2}\left|k W_{k}^{2}\right|+\frac{e^{2}}{2 \pi|k|} W_{k}\right)\left(1+\mathcal{O}\left(\frac{1}{|k|}\right)\right)$, hence $E_{0}$ is finite due to our assumptions (3b) on the potential.)

We now construct the unitary operator $\mathcal{U}$ implementing the Bogoliubov transformation (32), i.e.

$$
C(k)=\mathcal{U} c(k) \mathcal{U}^{*} \quad \forall k \in \Lambda^{*} .
$$

It is easy to see that operators $\mathcal{U}_{k}$ satisfying $C( \pm k)=\mathcal{U}_{k} c( \pm k) \mathcal{U}_{k}^{*}$ for all $k>0$ are given by

$$
\mathcal{U}_{k}=\mathrm{e}^{S_{k}}, \quad S_{k}=\lambda_{k}\left(c(k) c(-k)-c^{*}(k) c^{*}(-k)\right)
$$

which are unitary since the operators $S_{k}$ are screw-hermitian.

$$
\mathcal{U}=\mathrm{e}^{S}, \quad S=\sum_{k>0} S_{k}
$$

This operator $S$ can be shown to exist and defines an anti-selfadjoint operator if and only if

$$
\sum_{k>0}|k|\left|\lambda_{k}\right|^{2}<\infty
$$

and therefore $(\overline{37 \mathrm{~d}})$ is necessary and sufficient for the unitary operator $\mathcal{U}$ to exist. This latter condition is equivalent to the second one in (3b) and thus fulfilled by assumption. Note that

$$
\mathcal{U}^{*} H \mathcal{U}=\frac{2 \pi}{L} h_{0}+\frac{2 \pi}{L} \sum_{k>0}\left(\omega_{k}\left(c^{*}(k) c(k)+c^{*}(-k) c(-k)\right)-2 \eta_{k} \frac{L}{2 \pi}\right) \equiv H_{D}
$$

and therefore $\mathcal{U}$ is the unitary operator diagonalizing the non-zero modes of our Hamiltonian.

\subsection{Gauge invariant states}

By the gauge fixing above we reduced the Hilbert space from $\mathcal{H}$ to $\mathcal{H}_{\text {phys }}^{\prime}$ containing all states invariant under small gauge transformations, i.e. of the form $\mathrm{e}^{\mathrm{i} \alpha(x)}$ with $\alpha(L / 2)=\alpha(-L / 2)$. There are, however, still large gauge transformations present which are generated by $\mathrm{e}^{\mathrm{i} 2 \pi x / L}$. It is important to note that physical states need not be invariant under these latter transformations, but it is useful to construct states with simple transformation properties. This is the origin of the $\theta$-vacuum.

The large gauge transformation $\mathrm{e}^{\mathrm{i} 2 \pi x / L}$ acts on the fields as follows

$$
\begin{array}{rll}
\psi(x) & \stackrel{R}{\rightarrow} \mathrm{e}^{\mathrm{i} 2 \pi x / L} \psi(x)=\left(R_{+} R_{-}\right)^{-1} \psi(x)\left(R_{+} R_{-}\right), \\
e Y & \stackrel{R}{\rightarrow} e Y-1
\end{array}
$$

where $R_{ \pm}$are the implementers of $\mathrm{e}^{\mathrm{i} 2 \pi x / L}$ in the chiral sectors of the fermions and are discussed in detail in Appendix A. The large gauge transformation $R$ obviously generates

\footnotetext{
${ }^{5}$ i.e. $S_{k}$ is selfadjoint
} 
a group $\mathbb{Z}, n \rightarrow R^{n}$, and we denote this group as $\mathbb{Z}_{R}$. Our aim is to construct the states in $\mathcal{H}_{\text {phys }}$ which carry an irreducible representation of $\mathbb{Z}_{R}$ and especially the ground states of our model.

We start with recalling that the Fermion Fock space can be decomposed in sectors of different chiral charges $\hat{\rho}^{ \pm}(0)$,

$$
\mathcal{H}_{\text {Fermion }}=\bigoplus_{n_{+}, n_{-} \in \mathbb{Z}} \mathcal{H}^{\left(n_{+}, n_{-}\right)}
$$

where

$$
\mathcal{H}^{\left(n_{+}, n_{-}\right)}=\left\{\Psi \in \mathcal{H}_{\text {Fermion }} \mid \hat{\rho}^{ \pm}(0) \Psi=n_{ \pm} \Psi\right\}=R_{+}^{n_{+}} R_{-}^{-n_{-}} \mathcal{H}^{(0,0)}
$$

(for a more detailed discussion see Appendix A). Thus,

$$
\mathcal{H}_{\text {phys }}=\mathcal{L}^{2}(\mathbb{R}, \mathrm{d} Y) \otimes \mathcal{H}_{\text {Fermion }}^{\prime}
$$

where

$$
\mathcal{H}_{\text {Fermion }}^{\prime}=\bigoplus_{n \in \mathbb{Z}} \mathcal{H}^{(n,-n)}, \quad \mathcal{H}^{(n,-n)}=\left(R_{+} R_{-}\right)^{n} \mathcal{H}^{(0,0)}
$$

is the zero charge subspace of the Fermion Fock space and we use the Schrödinger representation for the physical degree of freedom $Y=\int_{\Lambda} \mathrm{d} x A_{1}(x) / 2 \pi$ of the photon field as discussed in the last subsection. $\mathcal{H}_{\text {phys }}$ can therefore be spanned by states

$$
\Psi(n)=\varphi\left(Y+\frac{n}{e}\right)\left(R_{+} R_{-}\right)^{n} \Psi, \quad \varphi \in \mathcal{L}^{2}(\mathbb{R}, \mathrm{d} Y), \Psi \in \mathcal{H}^{(0,0)}
$$

which, under a large gauge transformation (39), transform as

$$
\Psi(n) \stackrel{R}{\rightarrow} \Psi(n-1) .
$$

Thus the states transforming under an irreducible representation of $\mathbb{Z}_{R}$ are given by

$$
\Psi^{\theta}=\sum_{n \in \mathbb{Z}} \mathrm{e}^{\mathrm{i} \theta n} \Psi(n) \stackrel{R}{\rightarrow} \mathrm{e}^{\mathrm{i} \theta} \Psi^{\theta}
$$

It is easy to calculate the inner products of these states,

$$
<\Psi_{1}^{\theta}, \Psi_{2}^{\theta^{\prime}}>=2 \pi \delta_{2 \pi}\left(\theta-\theta^{\prime}\right)<\Psi_{1}, \Psi_{2}>_{\mathrm{F}}\left(\varphi_{1}, \varphi_{2}\right)_{\mathcal{L}^{2}}
$$

$\left(2 \pi \delta_{2 \pi}(\theta)=\sum_{n \in \mathbb{Z}} \mathrm{e}^{\mathrm{i} n \theta}\right.$, since $<\left(R_{+} R_{-}\right)^{n} \Psi_{1},\left(R_{+} R_{-}\right)^{m} \Psi_{2}>=\delta_{n, m}<\Psi_{1}, \Psi_{2}>_{\mathrm{F}} ;<\cdot, \cdot>_{\mathrm{F}}$ and $\langle\cdot, \cdot\rangle_{\mathcal{L}^{2}}$ are the inner products in $\mathcal{H}_{\text {Fermion }}$ and $\mathcal{L}^{2}(\mathbb{R}, \mathrm{d} Y)$, respectively). Thus the states $\Psi^{\theta}$ actually are not elements in $\mathcal{H}_{\text {phys }}$ (they do not have a finite norm).

In our calculation of Green functions below we find it useful to use the notation

$$
<\Psi_{1}^{\theta}, \Psi_{2}^{\theta}>_{\theta} \equiv<\Psi_{1}, \Psi_{2}>_{\mathrm{F}}\left(\varphi_{1}, \varphi_{2}\right)_{\mathcal{L}^{2}}
$$

which can be regarded as redefinition of the inner product using a simple multiplicative regularization (dropping the infinite term $2 \pi \delta_{2 \pi}(0)$ ).

We now construct the ground states of our model. As expected, the quantum mechanical variables $P, X$ (31a) describing the zero mode $h_{0}$ of the Hamiltonian have a simple representation on the $\theta$-states (43),

$$
\begin{gathered}
P \Psi^{\theta}=\sum_{n \in \mathbb{Z}} \mathrm{e}^{\mathrm{i} \theta n} 2 e\left(Y+\frac{n}{e}\right) \varphi\left(Y+\frac{n}{e}\right)\left(R_{+} R_{-}\right)^{n} \Psi, \\
X \Psi^{\theta}=\sum_{n \in \mathbb{Z}} \mathrm{e}^{\mathrm{i} \theta n} \frac{\mathrm{i}}{2 e} \frac{L}{2 \pi} \frac{\partial}{\partial Y} \varphi\left(Y+\frac{n}{e}\right)\left(R_{+} R_{-}\right)^{n} \Psi .
\end{gathered}
$$


Thus the ground states of $h_{0}$ annihilated by $C(0)$ are of the form (42a) with

$$
\varphi_{0}(Y)=\left(\frac{\pi}{4 e^{2} \alpha}\right)^{\frac{1}{4}} \exp \left(-\alpha(2 e Y)^{2}\right)
$$

where $\alpha=\frac{1}{L} \sqrt{\frac{\pi^{3}}{2 e^{2}}\left(1-W_{0}\right)}$, and the other eigenstates are the harmonic oscillator eigenfunctions $\varphi_{n} \propto C^{*}(0)^{n} \varphi_{0}$. From $C(k)=\mathcal{U} c(k) \mathcal{U}^{*}$ and $c(k) \Omega_{\mathrm{F}}=0$ it is clear that the ground state of all $h_{k>0}$ is $\mathcal{U} \Omega_{\mathrm{F}}$. We conclude that the ground states of our model obeying $H \Psi_{0}^{\theta}=L E_{0} \Psi_{0}^{\theta}$ are given by

$$
\Psi_{0}^{\theta}=\sum_{n \in \mathbb{Z}} \mathrm{e}^{\mathrm{i} \theta n} \varphi_{0}\left(Y+\frac{n}{e}\right)\left(R_{+} R_{-}\right)^{n} \mathcal{U} \Omega_{\mathrm{F}}
$$

\subsection{Gauge invariant Green functions}

The observables of our model now are operators on $\mathcal{H}_{\text {phys }}$ where $\int_{\Lambda} \mathrm{d} x A_{1}(x)$ is represented by $2 \pi Y$. We recall that the fully gauge invariant field operators are the $\chi$, (12), which are represented in the present gauge fixed setting by

$$
\chi_{\sigma}(x)=\mathrm{e}^{\mathrm{i} 2 \pi e Y(x-r) / L} \psi_{\sigma}(x) .
$$

These operators depend on the $r \in \Lambda$ chosen. Bilinears such as meson operators are, however, independent of $r$ and give rise to translational invariant equal time Green functions. Moreover, on the quantum level not only the Wilson line $W\left[A_{1}\right]$ (11) but actually even

$$
e \int_{\Lambda} \mathrm{d} x A_{1}(x)+\frac{1}{2} Q_{5} \equiv w\left[A_{1}\right]
$$

is gauge invariant (note that $W\left[A_{1}\right]=\mathrm{e}^{\mathrm{i} w\left[A_{1}\right]}$ ). This operator is represented by $e Y+\frac{1}{2} Q_{5}=$ $P / 2$ (cf. (31a)).

The gauge invariant equal time Green functions of the model are the ground state expectation values of products $(\cdots)$ of meson operators and functionals $F[P, X]$ of the zero mode operators $P, X$. Since we only consider $(\cdots)$ which are also invariant under large gauge transformations, the transition amplitudes $\left\langle\Psi_{1}^{\theta},(\cdots) \Psi_{2}^{\theta^{\prime}}\right\rangle$ are always proportional to $2 \pi \delta\left(\theta-\theta^{\prime}\right)$. Thus the Green functions we consider can be defined as

$$
\left\langle\Psi_{0}^{\theta}, F[P, X] \chi_{\sigma_{1}}^{*}\left(x_{1}\right) \chi_{\tau_{1}}\left(y_{1}\right) \cdots \chi_{\sigma_{N}}^{*}\left(x_{N}\right) \chi_{\tau_{N}}\left(y_{N}\right) \Psi_{0}^{\theta}\right\rangle_{\theta}
$$

(note that $\left\langle\Psi_{0}^{\theta}, \Psi_{0}^{\theta}\right\rangle_{\theta}=1$, cf. (45)).

Following [10] it is useful to define interacting fermion fields

$$
\Psi_{\sigma}(x)=\mathcal{U}^{*} \psi_{\sigma}(x) \mathcal{U}
$$

such that (50) becomes

$$
\text { Eq. }(50)=\left\langle\Omega^{\theta}, F[P, X] \Psi_{\sigma_{1}}^{*}\left(x_{1}\right) \Psi_{\tau_{1}}\left(y_{1}\right) \cdots \Psi_{\sigma_{N}}^{*}\left(x_{N}\right) \Psi_{\tau_{N}}\left(y_{N}\right) \Omega^{\theta^{\prime}}\right\rangle
$$

where

$$
\Omega^{\theta}=\sum_{n \in \mathbb{Z}} \mathrm{e}^{\mathrm{i} \theta n} \varphi_{0}\left(Y+\frac{n}{e}\right)\left(R_{+} R_{-}\right)^{n} \Omega_{\mathrm{F}}
$$


is the $\theta$-state constructed from the free fermion vacuum.

The strategy to calculate Green functions of the model using bosonization techniques is the following: the relation (A2) of appendix A can be used to move the operators $R_{ \pm}$ and combine them to some power of $\left(R_{+} R_{-}\right)$. The operators $Q_{ \pm}$when applied to physical states become simple $\mathbb{C}$-numbers: $Q_{ \pm}\left(R_{+} R_{-}\right)^{n}=\left(R_{+} R_{-}\right)^{n}\left( \pm n+Q_{ \pm}\right)$for all integers $n$, and $Q_{ \pm} \Omega_{\mathrm{F}}=0$. For the exponentials of boson operators we use the decomposition into creation and annihilation parts outlined in A.4. The normal ordering procedure gives a product of exponentials of commutators which are (C-number) functions. For the correlation functions of meson operators $\chi_{\sigma}^{*}(x) \chi_{\sigma^{\prime}}(y)$ we obtain:

$$
\begin{aligned}
& \left\langle\Psi_{0}^{\theta}, \chi_{ \pm}^{*}(x) \chi_{ \pm}(y) \Psi_{0}^{\theta}\right\rangle_{\theta}=\mathrm{e}^{-\frac{\pi}{4 L} m(x-y)^{2}} \mathrm{e}^{\Delta(x-y)} g_{0}^{ \pm}(x-y) \\
& \left\langle\Psi_{0}^{\theta}, \chi_{ \pm}^{*}(x) \chi_{\mp}(y) \Psi_{0}^{\theta}\right\rangle_{\theta}=\mathrm{e}^{\mp \mathrm{i} \theta} \mathrm{e}^{-i \frac{2 \pi}{L}(x-y)} \mathrm{e}^{-\frac{\pi m}{4 L}\left((x-y)+\frac{2}{m}\right)^{2}} C(L) \mathrm{e}^{D(x-y)} .
\end{aligned}
$$

with

$$
\begin{aligned}
\Delta & =\sum_{k>0} \frac{2 \pi}{L k} \sinh ^{2}\left(\lambda_{k}\right)\left[\mathrm{e}^{\mathrm{i} k x}+\mathrm{e}^{-\mathrm{i} k x}-2\right], \\
D(x) & =-\sum_{k>0} \frac{\pi}{L k} \sinh \left(2 \lambda_{k}\right)\left[\mathrm{e}^{\mathrm{i} k x}+\mathrm{e}^{-\mathrm{i} k x}-2\right], \\
C(L) & =\frac{1}{L} \exp \left[\sum_{k>0} \frac{2 \pi}{k L}\left(\sinh \left(2 \lambda_{k}\right)-2 \sinh ^{2}\left(\lambda_{k}\right)\right)\right] .
\end{aligned}
$$

where $g_{0}^{ \pm}(x)=\frac{1}{L} \frac{e^{\mp i \frac{\pi}{L} x}}{1-e^{ \pm i \frac{2 \pi}{L}(x \pm i \varepsilon)}}$ is the 2-point function of free fermions, and the Schwinger mass is renormalized to $m^{2}=\frac{e^{2}}{\pi\left(1-W_{0}\right)}$.

Note that the Green function (52b) depends on $\theta$ and is non-zero due to chiral symmetry breaking as in the Schwinger model. As expected, for vanishing electromagnetic coupling, $e=0$, this Green function vanishes (due to the factor $\mathrm{e}^{-\pi / m L}$ appearing in (52b)).

From (52b) we can calculate the chiral condensate by setting $x=y$, and in the limit $L \rightarrow \infty$ we obtain

$$
\lim _{L \rightarrow \infty}\left\langle\Psi_{0}^{\theta}, \chi_{ \pm}^{*}(x) \chi_{\mp}(x) \Psi_{0}^{\theta}\right\rangle_{\theta}=\lim _{L \rightarrow \infty} \mathrm{e}^{\mp \mathrm{i} \theta} \mathrm{e}^{-\frac{\pi}{m L}} C(L)=\mathrm{e}^{\mp \mathrm{i} \theta} C
$$

with a constant $C$ which can be calculated in principle from eq. (53). In the special case of the Schwinger model $\left(W_{k}=0\right), C$ can be computed and we recover the well-known result $C_{W_{k}=0}=\frac{m}{4 \pi} \mathrm{e}^{\gamma}$ where $\gamma=0.577 \ldots$ is Eulers constant (see e.g. [18, 19]).

\section{Multiplicative regularization and the Thirring-Schwinger model}

We recall that the Thirring model is formally obtained from the Luttinger model in the limits

$$
L \rightarrow \infty, \quad V(x) \rightarrow g \delta(x)
$$

i.e. when the interaction becomes local and space becomes infinite. The first limit amounts to remove the IR cut-off of our model. By inspection it can be easily done in all Green 
functions. The second limit in (55) is non-trivial: we recall, that condition (3b) on the Luttinger potential requires sufficient decay of the Fourier modes $W_{k}$ of the interaction, and this is violated in the Thirring model where $W_{k}=W_{0}$ is independent of $k$. This latter condition was necessary for the interacting model to be well-defined on the Hilbert space of the non-interacting model.

A better understanding can be obtained by explicitly performing the limit (55) in the present setting. The idea is to find a family of Luttinger potentials $\left\{V_{\ell}(x)\right\}_{\ell>0}$ becoming local for $\ell \downarrow 0$, i.e. for all $\ell>0$ the condition (3b) is fulfilled and $\lim _{\ell \downarrow 0} V_{\ell}(x)=g \delta(x)$. Then for all $\ell>0$ everything is well-defined on the free Hilbert space and one can work out in detail how to regularize such that the correlation functions make sense for $\ell \downarrow 0$.

We note that a direct construction of the Thirring model in a framework similar to the one here has been completed in [5]. This construction seems to be, however, different from the one outlined below.

For the case of Luttinger-Schwinger model we split the function $\Delta(x)$ into a part corresponding to the pure Luttinger model and a part which describes the additional Schwinger coupling, i. e. $\Delta(x)=\left(\Delta(x)-\Delta^{e=0}(x)\right)+\Delta^{e=0}(x)$.

The limit $W_{k}=W_{0}=$ const. exists for $\Delta-\Delta^{e=0}$. As $L \rightarrow \infty$, the sum in (53) turns into an integral and we obtain

$$
\begin{aligned}
\Delta(x)-\Delta^{e=0}(x)= & \frac{1}{\sqrt{1-W_{0}^{2}}} \int_{0}^{\infty} \mathrm{d} k\left(\frac{1}{\sqrt{k^{2}+\mu^{2}}}-1\right)(\cos (k x)-1)+ \\
& \sqrt{\frac{1+W_{0}}{1-W_{0}}} \int_{0}^{\infty} \mathrm{d} k \frac{\mu^{2}}{k^{2}} \frac{1}{\sqrt{k^{2}+\mu^{2}}}(\cos (k x)-1) .
\end{aligned}
$$

The first integral becomes $K_{0}(|\mu x|)+\ln \frac{|\mu x|}{2}+\gamma$ and the expression in the last line is a second integral $(n=2)$ of $K_{0}$ defined iteratively by $\mathrm{Ki}_{n}(x)=\int_{x}^{\infty} \mathrm{Ki}_{n-1}(t) d t, \mathrm{Ki}_{0}=K_{0}$ [20]. Moreover we introduced a new mass by $\mu^{2}=e^{2} /\left(\pi\left(1+W_{0}\right)\right)$. Note that the singularities at the origin of the Bessel function are removed by the additional terms, consistent with $\Delta(0)=0$.

No regularization has been necessary so far. Renormalization comes along with $\Delta^{e=0}$. We choose a Luttinger-interaction such that $\left(1-W_{k}^{2}\right)^{-1 / 2}-1=2 a^{2} e^{-\ell k}$ where $\ell$ defines the range of the interaction. For this choice we find

$$
\Delta^{e=0}(x)=2 a^{2} \ln \left|\frac{\ell}{x+i \ell}\right|
$$

and obviously the Thirring limit makes sense only if one removes the singular part $\ln \ell$ which can be done by a wave function renormalization of the form

$$
\chi_{ \pm}(x) \rightarrow \tilde{\chi}_{ \pm}(x)=Z^{1 / 2}(a, \ell) \chi_{ \pm}(x) \quad \text { with } \quad Z^{1 / 2}(a, \ell)=\ell^{-a^{2}} .
$$

A similar discussion holds for the chirality mixing correlation function. The 2-point function of the Thirring-Schwinger model therefore become

$$
\begin{aligned}
\left\langle\Psi_{0}^{\theta}, \tilde{\chi}_{ \pm}^{*}(x) \tilde{\chi}_{ \pm}(0) \Psi_{0}^{\theta}\right\rangle_{\theta} & =e^{\Delta_{\mathrm{reg}}(x)} g_{0}^{ \pm}(x) \\
\left\langle\Psi_{0}^{\theta}, \tilde{\chi}_{ \pm}^{*}(x) \tilde{\chi}_{\mp}(0) \Psi_{0}^{\theta}\right\rangle_{\theta} & =\mathrm{e}^{\mp \mathrm{i} \theta} C_{\mathrm{reg}} \mathrm{e}^{D_{\mathrm{reg}}(x)}
\end{aligned}
$$


If we define $\tau_{0}$ by $\tanh \left(2 \tau_{0}\right)=W_{0}$ we can write

$$
\begin{aligned}
\Delta_{\mathrm{reg}}(x)= & \cosh \left(2 \tau_{0}\right)\left[K_{0}(|\mu x|)+\ln \frac{|\mu x|}{2}+\gamma\right]+ \\
& \frac{1}{2} e^{2 \tau_{0}}\left[1-\frac{\pi}{2}|\mu x|-\mathrm{Ki}_{2}(|\mu x|)\right]+\left(\cosh \left(2 \tau_{0}\right)-1\right) \ln |x|, \\
D_{\mathrm{reg}}(x)= & -\sinh \left(2 \tau_{0}\right)\left[K_{0}(|\mu x|)+\ln \frac{|\mu x|}{2}+\gamma\right]- \\
& \frac{1}{2} e^{2 \tau_{0}}\left[1-\frac{\pi}{2}|\mu x|-\mathrm{Ki}_{2}(|\mu x|)\right] \\
\ln C_{\mathrm{reg}}= & \gamma+\ln \frac{1}{2 \pi}+e^{-2 \tau_{0}} \ln \frac{\mu}{2} .
\end{aligned}
$$

We checked that all Green functions of the Thirring-Schwinger model have a well-defined limit after the wave function renormalization.

We would like to stress that this procedure can be naturally interpreted as low-energy limit of the Luttinger-Schwinger model: if one is interested only in Green functions describing correlations of far-apart fermions, the precise form of the Luttinger interaction $V(x)$ should be irrelevant and only the total interaction strength $g=\int \mathrm{d} x V(x)$ should matter. Thus as far as these correlators are concerned, they should be equal to the ones of the Thirring model corresponding to this coupling $g$.

\section{Conclusion}

We formulated and solved the Luttinger-Schwinger model in the Hamiltonian formalism. Structural issues like gauge invariance, the role of anomalies and the structure of the physical states were discussed in detail. The necessary tools for computing all equal time correlation functions were prepared and illustrated by calculating the 2-point Green functions. From this the chiral condensate and critical exponents were computed. We could also clarify how the non trivial short distance behavior of the Thirring-Schwinger model arises in a limit from the Luttinger-Schwinger model.

\section{Appendix A: Bosons from fermions and vice versa}

In this appendix we summarize the basics for the bosonization used in the main text to solve the Luttinger-Schwinger model. Bosonization is known in the physics literature since quite some time $([16,21,22,23])$, for a discussion of the older history see [10]).

We consider the fermion Fock space $\mathcal{H}_{\text {Fermion }}$ generated by the fermion field operators from the vacuum $\Omega_{\mathrm{F}}$ as described in the main text. We note that $\mathcal{H}_{\text {Fermion }}=\mathcal{H}_{+} \otimes \mathcal{H}_{-}$ where $\mathcal{H}_{ \pm}$are generated by the left- and right-handed chiral components $\hat{\psi}_{+}$and $\hat{\psi}_{-}$of our Dirac fermions. Bosonization can be formulated for the chiral components $\hat{\psi}_{ \pm}$separately as it leaves the two chiral sectors $\mathcal{H}_{ \pm}$completely decoupled. For our purpose it is more convenient to treat both chiral sectors together. 


\section{A.1 Structure of fermion Fock space}

We start by introducing two unitary operators $R_{ \pm}$which are defined up to an irrelevant phase factor (which we will leave unspecified) by the following equations,

$$
\hat{\psi}_{ \pm}(k) R_{ \pm}=R_{ \pm} \hat{\psi}_{ \pm}\left(k-\frac{2 \pi}{L}\right)
$$

and $R_{ \pm}$commutes with $\hat{\psi}_{\mp}$. A proof of existence and an explicit construction of these operators can be found in 24]. Here we just summarize their physical meaning and special properties.

It is easy to see that $R_{ \pm}$are just the implementors of Bogoliubov transformations given by the large gauge transformations $\psi_{ \pm}(x) \mapsto \mathrm{e}^{\mathrm{i} 2 \pi x / L} \psi_{ \pm}(x)$ and $\psi_{\mp}(x) \mapsto \psi_{\mp}(x)$, hence $R_{+} R_{-}$and $R_{+} R_{-}^{-1}$ implement the vector- and the axial large gauge transformations $\mathrm{e}^{\mathrm{i} 2 \pi x / L}$ and $\mathrm{e}^{\mathrm{i} \gamma_{5} 2 \pi x / L}$, respectively. These have non-trivial winding number 1 and change the vacuum $\Omega_{\mathrm{F}}$ to states containing (anti-) particles. The latter follows from the commutator relations with the chiral fermion currents

$$
\left(R_{ \pm}\right)^{-1} \hat{\rho}^{ \pm}(k) R_{ \pm}=\hat{\rho}^{ \pm}(k) \pm \delta_{k, 0} \cdot
$$

The essential point of bosonization is that the total Hilbert space $\mathcal{H}_{\text {Fermion }}$ can be generated from $\Omega_{\mathrm{F}}$ by the chiral fermion currents $\hat{\rho}^{ \pm}(k)$ and $R_{ \pm}$. More precisely, for all pairs of integers $n_{+}, n_{-} \in \mathbb{Z}$ we introduce the subspaces $\mathcal{D}^{\left(n_{+}, n_{-}\right)}$of $\mathcal{H}_{\text {Fermion }}$ containing all linear combinations of vectors

$$
\hat{\rho}^{+}\left(k_{1}\right) \cdots \hat{\rho}^{+}\left(k_{m_{+}}\right) \hat{\rho}^{-}\left(q_{1}\right) \cdots \hat{\rho}^{-}\left(k_{m_{-}}\right) R_{+}^{n_{+}} R_{-}^{-n_{-}} \Omega_{F}
$$

where $m_{ \pm} \in \mathbb{N}_{0}$ and $k_{i}, q_{i} \in \Lambda^{*}$. The basic result of the boson-fermion correspondence is the following

Lemma: The space

$$
\mathcal{D} \equiv \bigoplus_{n_{+}, n_{-} \in \mathbb{Z}} D^{\left(n_{+}, n_{-}\right)} .
$$

is dense in $\mathcal{H}_{\text {Fermion }}$ (for a proof see e.g. [16]).

Remark: This Lemma gives the following picture of the structure of the Fock space $\mathcal{H}_{\text {Fermion }}$ : It splits into superselection sectors $\mathcal{H}^{\left(n_{+}, n_{-}\right)}$(which are the closure of $\mathcal{D}^{\left(n_{+}, n_{-}\right)}$) containing the eigenstates of the chiral charges $Q_{ \pm}$with eigenvalues $n_{ \pm}$. The fermion currents $\hat{\rho}^{ \pm}(k)$ leave all these sectors invariant, and the operators $R_{ \pm}$intertwine different sectors, $R_{+}: \mathcal{H}^{\left(n_{+}, n_{-}\right)} \rightarrow \mathcal{H}^{\left(n_{+}+1, n_{-}\right)}$and $R_{-}: \mathcal{H}^{\left(n_{+}, n_{-}\right)} \rightarrow \mathcal{H}^{\left(n_{+}, n_{-}-1\right)}$.

\section{A.2 Kronig's identity}

The basic formula underlying the solution of our model is

$$
\hat{H}_{0}=\frac{\pi}{L}\left(Q_{+}^{2}+Q_{-}^{2}\right)+\frac{2 \pi}{L} \sum_{k>0}\left(\hat{\rho}^{+}(-k) \hat{\rho}^{+}(k)+\hat{\rho}^{-}(k) \hat{\rho}^{-}(-k)\right) .
$$

It expresses the free Dirac Hamiltonian in terms of bilinears of the chiral fermion currents.

\footnotetext{
${ }^{6}$ the w.n. of a smooth gauge transformation $\Lambda \rightarrow \mathrm{U}(1), x \mapsto \mathrm{e}^{\mathrm{i} \alpha(x)}$ is the integer $\frac{1}{2 \pi}(\alpha(L)-\alpha(0))$.
} 


\section{A.3 Boson-fermion correspondence}

The boson-fermion correspondence provides explicit formulas of the fermion operators $\psi_{ \pm}(x)$ in terms of operators $\hat{\rho}^{ \pm}(k)$ and $R_{ \pm}$,

$$
\psi_{ \pm}(x)=\lim _{\varepsilon \searrow 0} \psi_{ \pm}(x ; \varepsilon)
$$

(this limit can e.g. be understood in the weak sense for states in $\mathcal{D}$ ), where

$$
\psi_{ \pm}(x ; \varepsilon)=\frac{1}{\sqrt{L}} S_{ \pm}(x): \exp \left(K_{ \pm}(x ; \varepsilon)\right):
$$

with

$$
S_{ \pm}(x)=\mathrm{e}^{ \pm \mathrm{i} \pi x Q_{ \pm} / L}\left(R_{ \pm}\right)^{\mp 1} \mathrm{e}^{ \pm \mathrm{i} \pi x Q_{ \pm} / L}=\mathrm{e}^{\mp \pi \mathrm{i} x / L}\left(R_{ \pm}\right)^{\mp 1} \mathrm{e}^{ \pm \mathrm{i} 2 \pi x Q_{ \pm} / L}
$$

and

$$
K_{ \pm}(x ; \varepsilon)=\mp \frac{2 \pi}{L} \sum_{k \in \Lambda^{*} \backslash\{0\}} \frac{\hat{\rho}^{ \pm}(-k)}{k} \mathrm{e}^{-\mathrm{i} k x} \mathrm{e}^{-\varepsilon|k|}=-K_{ \pm}(x ; \varepsilon)^{*} .
$$

More explicitly, the normal ordering : $\cdots$ : is with respect to the fermion vacuum $\Omega_{\mathrm{F}}$ (cf. (20))

$$
: \exp \left(K_{ \pm}(x ; \varepsilon)\right):=\exp \left(K_{ \pm}^{(-)}(x ; \varepsilon)\right) \exp \left(K_{ \pm}^{(+)}(x ; \varepsilon)\right)
$$

where

$$
K_{ \pm}^{(\sigma)}(x ; \varepsilon)=\sigma \frac{2 \pi}{L} \sum_{k>0} \frac{\hat{\rho}^{ \pm}( \pm \sigma k)}{k} \mathrm{e}^{ \pm \sigma \mathrm{i} k x} \mathrm{e}^{-\varepsilon|k|}, \quad \sigma=+,-
$$

is such that $K_{ \pm}=K_{ \pm}^{(-)}+K_{ \pm}^{(+)}$and $K_{ \pm}^{(+)} \Omega_{\mathrm{F}}=0$ (cf. (20)).

\section{A.4 Interacting fermions}

From the definition of the interacting fermion fields $\Psi(x)$ (51a) and the representation of free fermions in terms of bosons, we are led to investigate the interacting kernel $\tilde{K}_{ \pm}(x)=$ $\mathcal{U}^{*} K_{ \pm}(x) \mathcal{U}:$

$$
\tilde{K}_{ \pm}(x)=\mp \frac{2 \pi}{L} \sum_{k \in \Lambda^{*} \backslash\{0\}} \frac{1}{k}\left(\cosh \left(\lambda_{k}\right) \hat{\rho}^{ \pm}(-k)-\sinh \left(\lambda_{k}\right) \hat{\rho}^{\mp}(-k)\right) \mathrm{e}^{-\mathrm{i} k x} \mathrm{e}^{-\varepsilon|k|} .
$$

It is convenient to write

$$
\tilde{K}_{ \pm}=\tilde{K}_{ \pm}^{(+)}+\tilde{K}_{ \pm}^{(-)}, \quad \tilde{K}_{ \pm}^{(\sigma)}=K c_{ \pm}^{(\sigma)}-K s_{\mp}^{(\sigma)}
$$

where the upper index refers to the creation- $(\sigma=-)$ and annihilation- $(\sigma=+)$ parts of operators and

$$
\begin{aligned}
K c_{ \pm}^{(\sigma)}(x) & =\sigma \sum_{k>0} \frac{2 \pi}{L k} \cosh \left(\lambda_{k}\right) \hat{\rho}^{ \pm}( \pm \sigma k) \mathrm{e}^{\mp \sigma \mathrm{i} k x} \mathrm{e}^{-\varepsilon k} \\
K s_{ \pm}^{(\sigma)}(x) & =\sigma \sum_{k>0} \frac{2 \pi}{L k} \sinh \left(\lambda_{k}\right) \hat{\rho}^{ \pm}( \pm \sigma k) \mathrm{e}^{\mp \sigma \mathrm{i} k x} \mathrm{e}^{-\varepsilon k}
\end{aligned}
$$


The nonzero commutators of these operators are

$$
\begin{aligned}
{\left[K c_{ \pm}^{(+)}(x), K c_{ \pm}^{(-)}(y)\right] } & =-\sum_{k>0} \frac{2 \pi}{L k} \cosh ^{2}\left(\lambda_{k}\right) \mathrm{e}^{\mp \mathrm{i} k(x-y)} \mathrm{e}^{-2 \varepsilon k} \\
{\left[K c_{ \pm}^{(+)}(x), K s_{ \pm}^{(-)}(y)\right] } & =-\sum_{k>0} \frac{\pi}{L k} \sinh \left(2 \lambda_{k}\right) \mathrm{e}^{\mp \mathrm{i} k(x-y)} \mathrm{e}^{-2 \varepsilon k} \\
{\left[K s_{ \pm}^{(+)}(x), K s_{ \pm}^{(-)}(y)\right] } & =-\sum_{k>0} \frac{2 \pi}{L k} \sinh ^{2}\left(\lambda_{k}\right) \mathrm{e}^{\mp \mathrm{i} k(x-y)} \mathrm{e}^{-2 \varepsilon k} .
\end{aligned}
$$

We find the following normal ordered expression for the interacting fermions

$$
\Psi_{ \pm}(x)=\frac{1}{\sqrt{L}} z S_{ \pm}(x): \mathrm{e}^{\tilde{K}_{ \pm}(x)}:
$$

where $z=\mathrm{e}^{-\sum_{k>0} \frac{2 \pi}{L k} \sinh ^{2}\left(\lambda_{k}\right)}$.

\section{Acknowledgments}

E.L. would like to thank the Erwin Schrödinger International Institute in Vienna for hospitality where part of this work was done, and the "Österreichische Forschungsgemeinschaft" for partial financial support in May/June 1994 when this work was begun. He would also like to thank S.G. Rajeev and Mats Wallin for usefull discussions. The authors thank the referee for valuable suggestions concerning the presentation of their results.

\section{References}

[1] Thirring W., Ann. Phys. 3, 91 (1958)

[2] Schwinger J., Phys. Rev. 128, 2425 (1962)

[3] Carey A. L. and Hurst A. C., Commun. Math. Phys. 801 (1981)

[4] Carey A. L. and Wright J. D., Rev. Math. Phys. 5, 551 (1993)

[5] Carey A. L., Ruijsenaars S. N. M., and Wright J. D., Commun. Math. Phys. 99347 (1985)

[6] Klaiber B., Quantum theory and statistical physics Vol. XA p141, Barut A. O. and Brittin W. E. (eds.), Lectures in Theoretical Physics, New York: Gordon \& Breach (1967)

[7] Lowenstein J. H. and Swieca J. A., Ann. Phys. 68, 172 (1970)

[8] Luttinger J. M., J. Math. Phys. 4, 1154 (1963)

[9] Mattis D. C. and Lieb E. H., J. Math. Phys. 6, 304 (1965)

[10] Heidenreich R., Seiler R., and Uhlenbrock D. A., J. Statist. Phys. 22, 27 (1980)

[11] Carey C. A. and Hannabuss K. C., J. Func. Anal. 75128 (1987) 
[12] Langmann E. and Semenoff G. W., Phys. Lett. B 341, 195 (1994)

[13] Manton N. S., Ann. Phys. 159, 220 (1985)

[14] Jackiw R., Topological investigations of quantized gauge theories. in: Relativity, Groups and Topology II, Les Houches 1983, DeWitt B. S. and Stora R. (eds.), North Holland, Amsterdam (1984)

[15] Grosse H. and Langmann E., Int. Jour. of Mod. Phys. 21, 5045 (1992)

[16] Carey A. L. and Ruijsenaars S. N. M., Acta Appl. Mat. 10, 1 (1987)

[17] Araki H, Publ. Res. Inst. Math. Sci. Kyoto 6, 385 (1970)

[18] Sachs I. and Wipf A., Hel. Phys. Acta 65, 652 (1992)

[19] Hetrick J. and Hosotani Y., Phys. Rev. D38, 2621 (1988)

[20] Abramowitz M., Stegun I., Handbook of mathematical functions, Dover publications, New York 1970

[21] Pressley A. and Segal G.: Loop Groups, Oxford Math. Monographs, Oxford (1986)

[22] Kac V. G. and Raina A. K.: Highest weight representations of infinite dimensional Lie algebras, World Scientific, Sinapore (1987)

[23] Mickelsson J.: Current Algebras and Groups, Plenum Monographs in Nonlinear Physics, Plenum Press (1989)

[24] Ruijsenaars S. N. M., J. Math. Phys. 18, 517 (1977)

[25] Gattringer C. and Seiler E., Ann. Phys. 233, 97 (1994)

[26] Sachs I., Wipf A., Ann. Phys. 249, 380 (1996),

Dettki A., Sachs I., and Wipf A., hep-th 9308067 